\title{
Low cost 3D global instability analysis and flow sensitivity based on dynamic mode decomposition and high-order numerical tools
}

\author{
Esteban Ferrer, ${ }^{* \dagger}$, Javier de Vicente and Eusebio Valero \\ ETSIA-UPM, School of Aeronautics, Plaza Cardenal Cisneros 3, E-28040 Madrid, Spain
}

\begin{abstract}
SUMMARY
We explore the recently developed snapshot-based dynamic mode decomposition (DMD) technique, a matrix-free Arnoldi type method, to predict 3D linear global flow instabilities. We apply the DMD technique to flows confined in an L-shaped cavity and compare the resulting modes to their counterparts issued from classic, matrix forming, linear instability analysis (i.e. BiGlobal approach) and direct numerical simulations. Results show that the DMD technique, which uses snapshots generated by a 3D non-linear incompressible discontinuous Galerkin Navier-Stokes solver, provides very similar results to classical linear instability analysis techniques. In addition, we compare DMD results issued from non-linear and linearised NavierStokes solvers, showing that linearisation is not necessary (i.e. base flow not required) to obtain linear modes, as long as the analysis is restricted to the exponential growth regime, that is, flow regime governed by the linearised Navier-Stokes equations, and showing the potential of this type of analysis based on snapshots to general purpose CFD codes, without need of modifications. Finally, this work shows that the DMD technique can provide three-dimensional direct and adjoint modes through snapshots provided by the linearised and adjoint linearised Navier-Stokes equations advanced in time. Subsequently, these modes are used to provide structural sensitivity maps and sensitivity to base flow modification information for 3D flows and complex geometries, at an affordable computational cost. The information provided by the sensitivity study is used to modify the L-shaped geometry and control the most unstable 3D mode. Copyright @ 2014 John Wiley \& Sons, Ltd.
\end{abstract}

\section{INTRODUCTION}

Over the last decades, flow instability analysis has arisen as a powerful method to understand the onset of flow bifurcations leading to unstable (e.g. unsteady, periodic or chaotic) flow regimes. During this time, various numerical techniques have been developed that are able to tackle instability problems of increasing complexity [1-5]. The simplest numerical techniques are concerned with local instability, where only a small region of the computational domain is selected for analysis. These spatially localised techniques (e.g. Orr-Sommerfeld equations) require some a priori knowledge of the flow behaviour in the surroundings of the studied region (e.g. parallel or weakly 
non-parallel flow). These techniques have proven very successful to provide insight into the flow physics governing flow instabilities $[4,6]$, but the application of local analysis to complex 3D flows remains limited [6].

Alternatively, the entire flow domain may be considered for analysis leading to a global instability analysis. These type of methods do not require an a priori knowledge of the local flow features and may be applied to complex geometries [2]. In particular, TriGlobal methods have been successful in predicting global flow instabilities for complex 3D configurations (e.g. see [7] for the first fully TriGlobal computation), wherever the computational effort can be handled [2]. These techniques rely on the numerical discretisation of the eigenvalue problem derived from considering the linearised Navier-Stokes (NS) equations around an equilibrium flow state: the base flow, which is generally steady (see Section 2). The resulting eigenvalue system may be large, and its solution may be expensive to compute (e.g. using an Arnoldi method coupled to a shift-inverse strategy [1]). This has been regarded as the main bottleneck for the extension of matrix forming methods to $3 \mathrm{D}$ flows and complex geometries. To alleviate the computational cost associated with performing a full three-dimensional eigenvalue calculation, the BiGlobal approach $[2,8]$ was introduced. In this simplification, the flow is assumed periodic in one of the three spatial directions, consequently reducing the computational cost; further details can be found in the following sections.

Alternatives to the solution of large eigenvalue systems (i.e. matrix forming methods) are provided by time-stepper techniques [9-12]. These type of approaches construct a Krylov subspace using snapshots issued from numerical simulations (or from experimental data). The resulting space can be subsequently analysed (e.g. Amoldi technique with $Q R$ orthogonalisation [13] or dynamic mode decomposition (DMD) [14]) to obtain flow instability information related to the underlying NS operator, as detailed in Section 2. These matrix-free methods enable computations of the most unstable eigenvalues without requiring the solution of a complete eigenvalue system as would require a matrix forming TriGlobal type analysis. Additionally, if the concern is the computation of transient growth type phenomena, time-steppers methods are often preferred. Examples of such analysis can be found in $[15,16]$ for boundary layers in the linear regime or in [17-19] in the non-linear cases.

Almost independently, post processing techniques, that enable the analysis of relevant flow structures either in terms of energy: Proper Orthogonal Decomposition or their dynamics: DMD, have been developed and applied to the analysis of laminar and turbulent flows [20, 21]. However, the application of the latter technique to the exponential growth regime (i.e. temporal flow region where linearisation of the NS equations holds) is still limited and is the focus of this paper. The recently developed DMD approach [22] enables the extraction of dynamic modes, which are spatial structures ranked by their dynamics. For this reason, the DMD technique provides very similar flow instability results when compared to BiGlobal analyses when applied to flow fields generated by linear operators and is equivalent to a time-stepping matrix-free method [10,12].

Let us note that linearised theories (e.g. matrix forming methods) can only predict flow instabilities in the exponential growth region (i.e. region where the linearised NS equations hold), whilst post processing techniques (e.g. DMD) can be applied to any flow region (snapshots produced by linearised or non-linear NS solvers), as far as the results are interpreted accordingly. However, in this work we will restrict our analysis to the exponential region. In addition, let us remark that if the non-linear equations are used to produce the snapshots, there is no need to consider any type of base flow assumption (e.g. steady state base flow is not required), since this is implicitly embedded in the simulation. Results obtained using the latter procedure can be found in following sections.

All these numerical methods aim to obtain relevant flow structures (i.e. direct global modes) and track their evolution. The evolution of such structures may be studied in a spatial or temporal framework [1], the latter being retained in this paper.

Global modes obtained from the adjoint NS equations (or linearised adjoint NS equations) lead to relevant structures for the adjoint system: adjoint global modes. The latter are essential to provide insight into flow control strategies [3,5]. In addition, with the direct and adjoint modes at hand, it is possible to compute the sensitivity of the flow to local feedback (or structural sensitivity) [23] and to base flow modifications [24]. These sensitivities relate to flow control strategies, since they 
provide information on the sensibility of modal growth rates to changes in the linearised system, see Section 4.

Finally, Direct Numerical Simulations (DNS) denote the numerical simulation of the NS equations without modelling or simplifications. Since instability analysis is concerned with the temporal evolution (i.e. growth or decay) of small perturbations superimposed upon a equilibrium state (i.e. the base flow), the level of accuracy demanded in the computations is very high. High-order numerical techniques (e.g. Spectral h/p or discontinuous Galerkin) are well suited to this purpose, since they provide numerical solutions where dissipative and dispersive errors are minimum [25]. The techniques used within this work (i.e. matrix forming BiGlobal, DNS and matrix-free methods) are all of high-order type and are detailed in Section 2.

From the overview of the various techniques available for global instability analysis, it becomes apparent that some degree of unification with respect to the applicability of these techniques is necessary. In addition, low cost techniques need to be available if stability analysis is to become an industrial engineering tool. To this aim, we present comparisons between matrix forming techniques BiGlobal and matrix-free DMD-based results. Namely, we include direct and adjoint modes extracted using these two methods. In addition, we compare DMD modes obtained from snapshots that have been generated from linearised, adjoint linearised and non-linear solvers. Finally, the low cost DMD method is used to obtain 3D sensitivity maps that provide valuable information to generate more stable modified geometries.

The following bullet points summarise the various aims of the present work:

- Show that the matrix-free DMD technique coupled to a linearised or a non-linear highorder DNS solver, provides very similar results to a classic matrix-forming linear instability analysis techniques (i.e. BiGlobal analysis), when analysing the exponential growth flow region. Additionally, we clarify the limits of usability and methodology for the application of snapshot-based methods.

- Show that the DMD technique provides global direct and adjoint modes, when considering the linearised and non-linear NS equations as long as the flow is not saturated. The possibility of using the DMD technique with non-linear solvers (i.e. no base flow required) should enable a wider range of applications. In addition, this paper shows that the DMD technique can provide instability results from an existing non-linear code without the necessity of writing a linearised version.

- Show that it is possible to obtain an approximation of the 3D direct and adjoint modes, as well as the associated sensitivity fields (e.g. to local feedback and to base flow modifications), at a reduced computational cost for general complex 3D geometries using DMD.

- Show that the obtained DMD modes compare very well with more classical, matrix forming, linear instability analysis techniques (i.e. BiGlobal analysis).

- Use the information obtained from the sensitivity maps to provide a modified L-shaped cavity where the most unstable 3D instability has lower growth rates than originally.

- Provide physical 3D global instability and sensitivity results for the L-shaped cavity, which have not been reported previously.

- Finally, we provide the first, to the authors' knowledge, results for direct, adjoint modes and sensitivity maps using a high-order incompressible discontinuous Galerkin DNS numerical solver.

This paper is structured as follows. In Section 2, we describe the various formulations (i.e. nonlinear, linearised and adjoint NS equations) and numerical methodologies (i.e. DNS, BiGlobal and DMD) used to obtain direct and adjoint modes. Then, we describe in Section 3 the L-shaped cavity problem and the flow conditions for the analysis. Results for the direct global modes and comparisons between matrix forming and matrix-free methods are also presented in Section 3, whilst adjoint modes and sensitivity results are included in Section 4. Finally in Section 5, a modified geometry for the L-shaped is presented, where the information provided by the sensitivity maps has been used to reduce the growth rate of the most unstable 3D mode. 


\section{NUMERICAL TECHNIQUES}

This section describes the three methodologies used to predict and analyse flow instabilities. We limit the scope of this analysis to the incompressible NS equations, which are described hereafter.

Let $\Omega$ be a domain in $\mathbb{R}^{d}$, where $d=2,3$ represents the problem dimension with boundaries $\partial \Omega$, and associated outward unit normal vector $\mathbf{n}$. Domain boundaries may be of Dirichlet $\left(\partial \Omega_{D}\right)$ or Neumann $\left(\partial \Omega_{N}\right)$ type, where $\partial \Omega=\partial \Omega_{D} \cup \partial \Omega_{N}$ and $\partial \Omega_{D} \cap \partial \Omega_{N}=\emptyset$. The unsteady nondimensional incompressible NS equations read:

$$
\begin{aligned}
\frac{\partial \mathbf{u}}{\partial t}+\mathbf{N}(\mathbf{u}) & =-\nabla p+\frac{1}{\operatorname{Re}} \nabla^{2} \mathbf{u} & & \text { in } \Omega \times[0, T], \\
\nabla \cdot \mathbf{u} & =0 & & \text { in } \Omega \times[0, T], \\
\mathbf{u}(t=0) & =\mathbf{u}_{\mathbf{0}} & & \text { in } \Omega, \\
\mathbf{u} & =\mathbf{L}_{\mathbf{D}} & & \text { on } \partial \Omega_{D} \times[0, T], \\
\frac{1}{\operatorname{Re}} \frac{\partial \mathbf{u}}{\partial \boldsymbol{n}}-p \mathbf{n} & =\mathbf{0} & & \text { on } \partial \Omega_{N} \times[0, T],
\end{aligned}
$$

where $t$ represents the dimensionless time, $\mathbf{u}=(u, v, w)^{T}$ and $p$ are the non-dimensionalised velocity vector and static pressure (normalised using upstream dynamic pressure $q=\varrho U^{2}$, where $\varrho$ is the fluid density and $U$ represents a characteristic velocity) respectively and Re is the Reynolds number (i.e. $\operatorname{Re}=U L / v$ where $L$ is a characteristic length scale and $v$ is the kinematic viscosity). In addition, $\mathbf{u}_{\mathbf{0}}$ represents an initial condition for the velocity field and $\mathbf{L}_{\mathbf{D}}$ defines the Dirichlet data for the velocity. The term $\mathbf{N}(\mathbf{u})$ represents the non-linear contributions which can be, without loss of generality, expressed in their convective form: $\mathbf{N}(\mathbf{u})=(\mathbf{u} \cdot \nabla) \mathbf{u}$.

Linearisation of the NS equations can be accomplished by decomposing the velocity solutions into a base flow component $\overline{\mathbf{u}}$ (generally assumed steady i.e. $\frac{\partial \overline{\mathbf{u}}}{\partial t}=\mathbf{0}$ or time periodic as in Floquet type analysis $[1,26])$ and a relatively small perturbation field $\mathbf{u}^{\prime}$, such that the velocity field can be reconstructed using summation: $\mathbf{u}=\overline{\mathbf{u}}+\mathbf{u}^{\prime}$. In addition, the pressure field is decomposed similarly: $\mathbf{p}=\overline{\mathbf{p}}+\mathbf{p}^{\prime}$

Substituting these decompositions into the NS equations Equation (1), and subtracting the base flow equation, we obtain the following system for the perturbation field:

$$
\begin{aligned}
\frac{\partial \mathbf{u}^{\prime}}{\partial t}+\mathbf{L}\left(\overline{\mathbf{u}}, \mathbf{u}^{\prime}\right) & =-\nabla p^{\prime}+\frac{1}{\operatorname{Re}} \nabla^{2} \mathbf{u}^{\prime} & & \text { in } \Omega \times[0, T], \\
\nabla \cdot \mathbf{u}^{\prime} & =0 & & \text { in } \Omega \times[0, T], \\
\mathbf{u}^{\prime}(t=0) & =\mathbf{u}_{\mathbf{0}}^{\prime} & & \text { in } \Omega, \\
\mathbf{u}^{\prime} & =\mathbf{0} & & \text { on } \partial \Omega_{D} \times[0, T], \\
\frac{1}{\operatorname{Re}} \frac{\partial \mathbf{u}^{\prime}}{\partial \mathbf{n}}-p^{\prime} \mathbf{n} & =\mathbf{0} & & \text { on } \partial \Omega_{N} \times[0, T],
\end{aligned}
$$

where the term $\mathbf{L}\left(\overline{\mathbf{u}}, \mathbf{u}^{\prime}\right)=(\overline{\mathbf{u}} \cdot \nabla) \mathbf{u}^{\prime}+\left(\mathbf{u}^{\prime} \cdot \nabla\right) \overline{\mathbf{u}}$ represents the linearised advective terms, where the quadratic contributions for the fluctuating velocities (i.e. $\left(\mathbf{u}^{\prime} \cdot \nabla\right) \mathbf{u}^{\prime}$ ) have been neglected (i.e. assumed small when compared to the retained terms $\left.\mathbf{L}\left(\overline{\mathbf{u}}, \mathbf{u}^{\prime}\right)\right)$. In addition, the Reynolds number in the linearised equations is defined as $\operatorname{Re}=U L / \nu \approx \bar{U} L / \nu$, for a mean characteristic base flow $\bar{U}$. Further details may be found in [1] or [25].

Finally, the linearised adjoint NS equations can be derived by considering the time-space $L^{2}$ inner product (i.e. $\left.\left\langle\mathbf{q}^{\prime}, \mathbf{q}^{*}\right\rangle=\int_{0}^{T} \int_{\Omega} \mathbf{q}^{\prime} \mathbf{q}^{*} d \mathbf{x} d t\right)$ between the linearised NS $\left(L^{N S}\right.$ ) equation and the linearised adjoint NS $\left(L_{A d j}^{N S}\right)$ equations such that: $\left\langle L^{N S} \mathbf{q}^{\prime}, \mathbf{q}^{*}\right\rangle-\left\langle\mathbf{q}^{\prime}, L_{A d j}^{N S} \mathbf{q}^{*}\right\rangle=\mathbf{0}$, where 
$\mathbf{q}^{\prime}=\left(\mathbf{u}^{\prime}, p^{\prime}\right)^{T}$ denotes the direct modes and $\mathbf{q}^{*}=\left(\mathbf{u}^{*}, p^{*}\right)^{T}$ the adjoint counterparts. The resulting linearised adjoint NS equations read:

$$
\begin{aligned}
-\frac{\partial \mathbf{u}^{*}}{\partial t}+\mathbf{L}_{\mathrm{Adj}}\left(\overline{\mathbf{u}}, \mathbf{u}^{*}\right) & =-\nabla p^{*}+\frac{1}{\operatorname{Re}} \nabla^{2} \mathbf{u}^{*} & & \text { in } \Omega \times[0, T], \\
\nabla \cdot \mathbf{u}^{*} & =0 & & \text { in } \Omega \times[0, T], \\
\mathbf{u}^{*}(t=T) & =\mathbf{u}^{\prime} \mathbf{T} & & \text { in } \Omega, \\
\mathbf{u}^{*} & =\mathbf{0} & & \text { on } \partial \Omega_{D} \times[0, T], \\
(\overline{\mathbf{u}} . \mathbf{n}) \mathbf{u}^{*}+\frac{1}{\operatorname{Re}} \frac{\partial \mathbf{u}^{*}}{\partial \boldsymbol{n}}-p^{*} \mathbf{n} & =\mathbf{0} & & \text { on } \partial \Omega_{N} \times[0, T],
\end{aligned}
$$

where $\mathbf{L}_{\text {Adj }}\left(\overline{\mathbf{u}}, \mathbf{u}^{*}\right)=-(\overline{\mathbf{u}} \cdot \nabla) \mathbf{u}^{*}+(\nabla \overline{\mathbf{u}})^{T} \mathbf{u}^{*}$. By setting the time $t$ to a negative time $-\bar{t}$, where $\bar{t}$ denotes a new time variable, one can use the numerical scheme defined to integrate the equations forward in time $[27,28]$ to solve the adjoint equations as noted previously by Barkley et al. [13].

\subsection{Direct numerical simulations: non-linear, linearised and adjoint linearised solvers}

Flow solutions of the non-linear 3D incompressible NS equations, are obtained from the unsteady high-order (order $\geq 3$ ) $h / p$ Discontinuous Galerkin (DG) - Fourier solver developed by the first author and detailed in [27-29]. This high-order solver provides highly accurate solutions on static and moving meshes composed of mixed triangular-quadrilateral meshes and can cope with curved boundary elements. A second order stiffly stable method is used to discretise in the NS equations in time whilst spatial discretisation is provided by the DG - Symmetric Interior Penalty Galerkin formulation with modal basis functions in the $x-y$ plane. Spatial discretisation in the $z$-direction is provided by a purely spectral method that uses Fourier series and allows computation of spanwise periodic three-dimensional flows. The solver has been widely validated for a variety of flows, including bluff body flows, airfoil and blade aerodynamics under static and rotating conditions $[27,28]$.

A linearised version of the previous code may be obtained via modification of the routines to compute the non-linear terms (i.e. $\mathbf{N}(\mathbf{u}) \longrightarrow \mathbf{L}\left(\overline{\mathbf{u}}, \mathbf{u}^{\prime}\right)$ ) and modification of boundary conditions (i.e. $\mathbf{L}_{\mathbf{D}} \longrightarrow \mathbf{0}$ ). In addition, a linearised adjoint DNS solver has been obtained through the modification of the non-linear terms (i.e. $\mathbf{N}(\mathbf{u}) \longrightarrow \mathbf{L}_{\mathbf{A d j}}\left(\overline{\mathbf{u}}, \mathbf{u}^{*}\right)$ ) and boundary conditions. Results issued from this solver for the direct solutions are reported in Section 3 and in Section 4 for the time advancement of the adjoint equations.

Finally, when computing cavity flows, no-slip Dirichlet boundary conditions are imposed at walls except for the lid driving wall, where $\mathbf{u}=(1,0,0)^{T}$ is defined. To avoid corner singularities, in previous work ([8]), the boundary condition for the lid velocity is regularised, when continuous high-order numerical methods based on the strong form of the governing equations. However, when using a weak imposition of boundary conditions, as enabled by the weak form of the DG formulation [28], we found that no regularisation is necessary. All computations included in this work have been performed with polynomial orders $k=17$ per triangular mesh element $\left(N^{e l}=6\right)$ and used 64 Fourier planes $\left(F F T_{z}=64\right)$ to discretise the $z$-direction, providing a total number of degrees of freedom (i.e. $D O F=N^{e l} \frac{1}{2}(k+1)(k+2) F F T_{z}$ ) of 65.664 (1026 per Fourier plane). Finally, all computations have been obtained using a second order time advancement scheme with a nondimensional time step $\Delta t=0.001$.

\subsection{BiGlobal linear instability analysis}

BiGlobal instability analysis $[2,8]$ is a matrix forming technique that assumes homogeneity in one spatial direction, reducing the computational cost of performing a full 3D TriGlobal instability analysis. Since the linearised version of the NS equations does not couple modes through the non-linear terms, it is possible to solve an independent system for each spanwise wavenumber $\beta$ 
of the perturbations instead of solving a coupled 3D system. Consequently, this approach is appropriate for the analysis of the temporal evolution of small-amplitude perturbations, superimposed upon a steady two-dimensional equilibrium state (i.e. the base flow), as shown previously for the linearised NS equations. In the BiGlobal approach, the velocity and pressure fields are decomposed as $\mathbf{q}(x, y, z, t)=\overline{\mathbf{q}}(x, y)+\mathbf{q}^{\prime}(x, y, t)=\overline{\mathbf{q}}(x, y)+\hat{\mathbf{q}}(x, y) e^{i(\beta z-\lambda t)}+c . c$. , where $\beta=2 \pi / L_{z}$ denotes a characteristic wavenumber in the $z$ direction, with corresponding physical periodic wavelength $L_{z}$. In addition, $\lambda=\left(\lambda_{r}, \lambda_{i}\right)$ is a complex number whose real and imaginary parts represent the perturbation growth rate and frequency, respectively and $\hat{\mathbf{q}}(x, y)$ denotes the modal shape (i.e. eigenvector) associated to a particular pair of $\beta$ and $\lambda$. Substitution of the perturbation $\mathbf{q}^{\prime}$ into the linearised NS equations, Equation (2), results in an 2D eigenvalue problem (for a fixed spanwise wavenumber $\beta$ ) which can be solved to obtain linear instability eigenmodes with associated complex eigenvalues $(\lambda)$ providing dynamic information of the perturbed flow.

The BiGlobal solver relies on a high-order continuous (i.e. $C^{1}$ ) multi-domain Chebyshev spectral collocation technique. Implementation details and validation can be found in [8]. BiGlobal computations included in this work used quadrilateral elements and required polynomials of order $k=20$. The resulting number of degrees of freedom for a $2 \mathrm{D}$ domain with a fixed spanwise wavenumber $\beta$, is 1200 (i.e. $D O F=N^{e l} k^{2}$ ).

Before continuing, we summarise various assumptions used throughout the development of the BiGlobal approach. Firstly, it is required that an equilibrium state, i.e. the base flow, exists. This requires a steady state solution for the two-dimensional case, which in turn serves as an input for the instability calculation. Secondly, the modelled perturbation is required to be periodic in the $z$ direction. Finally, the flow regime is assumed linear and hence non-linearities in the saturated flow are beyond its scope.

\subsection{Dynamic mode decomposition: snapshot analysis}

In recent years, the snapshot-based technique DMD, has seen an increased popularity $[22,30]$ and has been applied to a variety of flows including numerical and experimental data, e.g. [14, 21, 31, 32]. The formulation retained here can be found in [22], but other algorithms have been proposed $[30,33]$. In addition, we note that the temporal framework for the study of flow instability is retained, but that the DMD with spatial snapshots is also possible [22].

It was shown by Mezic [21], Rowley et al. [31] and more recently by Bagheri [14] that the modes resulting from the DMD algorithm are approximations of Koopman modes. Koopman operators are infinite dimensional linear operators capable of describing non-linear processes. Some of the associated infinite number of modes related to this operator may be approximated through the DMD decomposition [21]. In the case of the approximated operator being linear (exponential growth region), we will show that the DMD results are very similar to classical matrix forming global approaches for stability analysis.

Schmid [22] described in detail the DMD technique and hence only a summary of the algorithm is described here. Given a sequence of 1 to $N$ flowfield snapshots (e.g. taking one or all variables of the flow field), one can construct the following matrix:

$$
\mathbf{V}_{1}^{N}=\left\{\mathbf{v}\left(\mathbf{t}_{1}\right), \mathbf{v}\left(\mathbf{t}_{2}\right), . ., \mathbf{v}\left(\mathbf{t}_{\mathbf{N}}\right)\right\}
$$

where subindex and superindex denote the first and last values of the sequence, respectively. Let us note that this data needs to be ordered, and that the snapshots require a constant sampling time $\Delta \tau$ such that: $t_{j+1}=t_{j}+\Delta \tau$ for all $j=1, \ldots, N$. In the case of linear stability analysis and within the exponential growth region, one can define a linear operator $\mathbf{A}$ (i.e. a numerical approximation of the linearised NS operator) between snapshots such that $v\left(t_{j+1}\right)=\mathbf{\Lambda} v\left(t_{j}\right)$, and rewrite Equation (4) as a Krylov sequence [34]:

$$
\mathbf{V}_{1}{ }^{N}=\left\{\mathbf{v}\left(\mathbf{t}_{1}\right), \mathbf{A v}\left(\mathbf{t}_{1}\right), \ldots, \mathbf{A}^{N-1} \mathbf{v}\left(\mathbf{t}_{1}\right)\right\}
$$


It is easy to see that for an ordered sequence, Equation (5) can be equated to Equation (4), to lead:

$$
\mathbf{A}\left\{\mathbf{v}\left(\mathbf{t}_{1}\right), \mathbf{v}\left(\mathbf{t}_{2}\right), \ldots, \mathbf{v}\left(\mathbf{t}_{\mathbf{N}-1}\right)\right\}=\left\{\mathbf{v}\left(\mathbf{t}_{2}\right), \mathbf{v}\left(\mathbf{t}_{3}\right), \ldots, \mathbf{v}\left(\mathbf{t}_{\mathbf{N}}\right)\right\}
$$

which can be written in matrix form as:

$$
\mathbf{A V}_{1}{ }^{N-1}=\mathbf{V}_{2}{ }^{N}
$$

The algorithm continues by obtaining the Singular Value Decomposition (SVD) of the matrix $\mathbf{V}_{1}{ }^{N-1}=\mathbf{U} \Sigma \mathbf{W}^{H}$, where the superscript $H$ denotes the conjugate transpose. Replacing the SVD definition into Equation (7), leads to $\mathbf{A} \mathbf{U} \Sigma \mathbf{W}^{H}=\mathbf{V}_{2}{ }^{N}$. To find the reduced matrix $\widetilde{S}$ associated to the initial system described by $\mathbf{A}$, it suffices to rewrite the previous equality as:

$$
\widetilde{S}=\mathbf{U}^{H} \mathbf{A} \mathbf{U}=\mathbf{U}^{H} \mathbf{V}_{2}{ }^{N} \mathbf{W} \mathbf{\Sigma}^{-1} .
$$

Inspection of Equation (8) reveals that the reduced matrix $\widetilde{S}$ is the projection of the matrix A onto the Proper Orthogonal Decomposition space contained in $\mathbf{U}$, and obtained through the singular value decomposition [22].

Having found the reduced matrix $\widetilde{S}$, one can obtain the reduced DMD modes $\mathbf{y}_{i}$ and associated eigenvalues $\mu_{i}$ (i.e. growth rates $\operatorname{Re}\left(\mu_{i}\right)$ and frequencies $\operatorname{Im}\left(\mu_{i}\right)$ mapped to the unit circle) of the reduced system by solving for the eigenvalues of $\widetilde{S} \mathbf{y}_{i}=\mu \mathbf{y}_{i}$. One can then recover the approximated eigenmodes of the matrix $\mathbf{A}$ by projecting into the original space using $\phi_{i}=\mathbf{U y}_{i}$. To retrieve the growth rates and frequencies in the complex half-plane, one can map the eigenvalues using: $\lambda_{i}=\log \left(\mu_{i}\right) / \Delta \tau$.

The numerical convergence of the DMD technique is dictated by the sampling frequency $f_{D M D}=1 / \Delta \tau$, where $\Delta \tau$ represents the sampling time between snapshots extracted from the DNS computation. On the one hand, to capture the highest frequency within the analysed flow, it is required that $f_{D M D} \geq 2 f_{\text {flow }}$, where $f_{\text {flow }}$ is the frequency of the flow feature to be captured and the factor of two is dictated by Nyquist criterion. In addition, note that if the flow frequency is not known a priori, then we select a small sampling time (high frequency) to cover most of the flow spectrum and avoid aliasing. On the other hand, the number of necessary snapshots (to obtain unchanged eigenvalues) is a priori unknown and hence for each case we perform tests where we increase the number of snapshots until convergence is reached, in terms of the most unstable eigenvalues. Let us note that the DMD technique provides valuable information whenever the flow exhibits distinct frequencies, but its applicability is limited when analysing flows that show broadband spectrums.

We finalise by noting some of the advantages of the DMD algorithm detailed in this section. This algorithm enables the post-process of only a limited flow region, which reduces drastically the computational cost for the extraction of the eigenmodes and related dynamical information [22]. In addition, the algorithm does not require all flow variables to be considered for the analysis, indeed, most of the results presented in this work use only one variable ( $w$-velocity component for the Lshaped cavity). The latter enables the reduction of the computational cost by a factor of three of the original 3D vector field. Finally, no shift and inverse type of strategy has been required in this work to obtain accurate eigenmodes, which has been shown to be necessary when using other matrix-free Arnoldi type algorithms $[2,12]$. The shift and invert technique is sometimes necessary to extract modes whose eigenvalues are close to the unit circle (e.g. most unstable eigenvalues associated to flows near bifurcations). It may therefore be concluded that enhanced robustness can be achieved when compared to more traditional matrix-free Arnoldi methods.

To summarise, the DMD method is a robust technique that provides accurate modes and dynamical information at a reduced computational cost, by reducing both the spatial region of analysis and the number of variables required to obtain qualitative and quantitative information. In addition, it can be applied to numerical or experimental data providing dynamical information for linear and non-linear flows. 


\subsection{Summary of methods and notation}

Henceforth and to simplify the notation, we denote $\mathcal{N} D N S+D M D$, the combination of producing snapshots using the full non-linear NS solver and subsequently analysing these flow fields using the described DMD technique. In addition, $\mathcal{L}$ DNS+DMD, will denote the combination of producing snapshots using the linearised version of the NS solver combined with the DMD analysis.

\section{DIRECT PROBLEM: L-SHAPED CAVITY AND THREE-DIMENSIONAL INSTABILITIES}

Cavity flows are of major interest both for their simplicity of analysis and because they may be regarded as a simplified version of geometries found in industrial applications, e.g. airplane landing gears compartments or short-dwell coaters [35].

Let us first clarify the scope of the various techniques described in previous sections. To this end, we introduce a DNS simulation of a L-shaped cavity at $\mathrm{Re}=900$. Figure 1 .(a) shows the $2 \mathrm{D}$ steady base flow (streamlines and velocity magnitude contours) resulting from driving the flow inside a Lshaped cavity with a lid velocity on the upper wall. In addition, Figure 1.(b) depicts the evolution of the logarithm of the absolute value of the maximum $w$-velocity in the cavity as a function of time obtained using the discontinuous Galerkin DNS solver. In the latter plot, two zones can be clearly identified: an exponential growth regime (coloured red on the left) and a saturated regime (coloured blue on the right). These two flow regions correspond to zones where the linearised NS or the non-linear NS equations describe the flow behaviour. The red curve may be simulated by means of a linearised or a non-linear DNS solver, but flow character in the saturated region can only be captured through a non-linear DNS solver. We will now compare the various techniques presented, which are used for the analysis of the L-shaped cavity problem. We utilise this geometry to asses the scope and limits of each numerical approach and to provide qualitative and quantitative instability results.

The flow features developing within this geometry have been studied numerically by other authors $[36,37]$ and the 2D bifurcations by $[38,39]$ but to the authors' knowledge, there has not been any 3D instability analysis performed in this geometry, other than the study included in de Vicente's $\mathrm{PhD}$ dissertation [8] (second author of this paper). Cavity flows are characterised by 3D instabilities developing at lower Reynolds numbers (on the basis of the geometrical cavity length and the lid velocity) than 2D instabilities [40-42]. For this reason, they are ideal candidates for testing new tools for analysis of $3 \mathrm{D}$ flow structures. It is worth mentioning that the underlying bifurcation mechanism
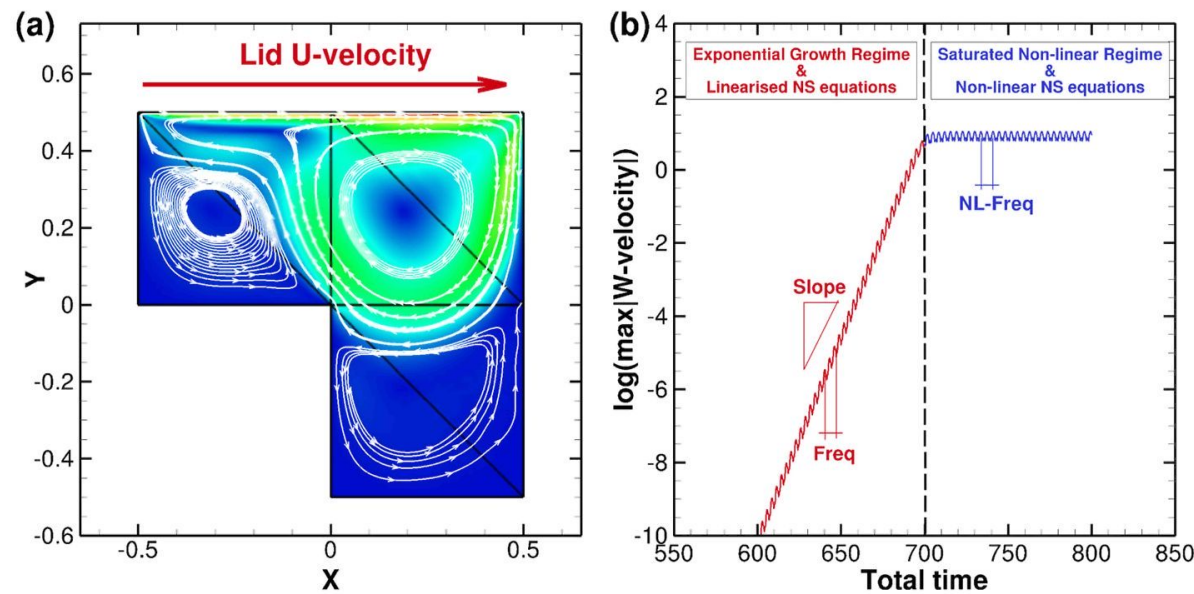

Figure 1. L-shaped cavity at Reynolds 900 and non-dimensional spanwise length of $L_{z}=0.628$. Results obtained from non-linear DNS computation: polynomial order $\mathrm{k}=17$ in the $\mathrm{x}-\mathrm{y}$ plane and 64 Fourier planes in the $z$-direction. (a) Velocity contours [0:1] and streamlines showing the 2D flow pattern and (b) Time evolution of the non-dimensional maximum $w$-velocity component ( $z$-direction), colours show exponential growth regime (red) and saturated non-linear regime (blue). 


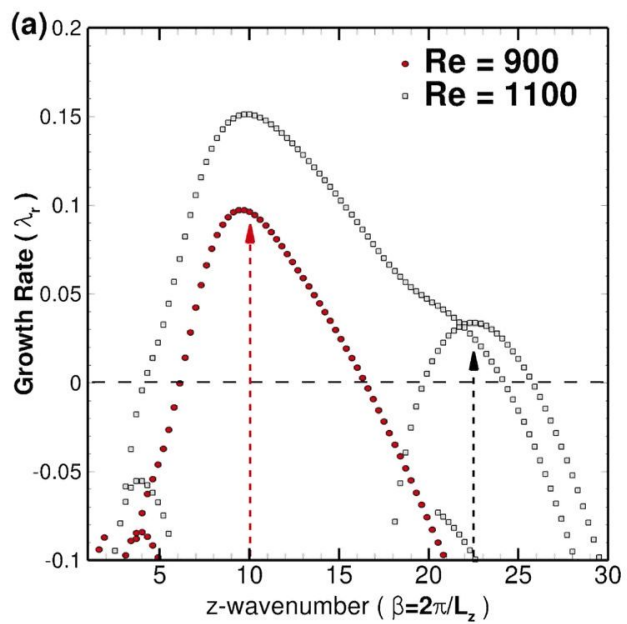

(b)

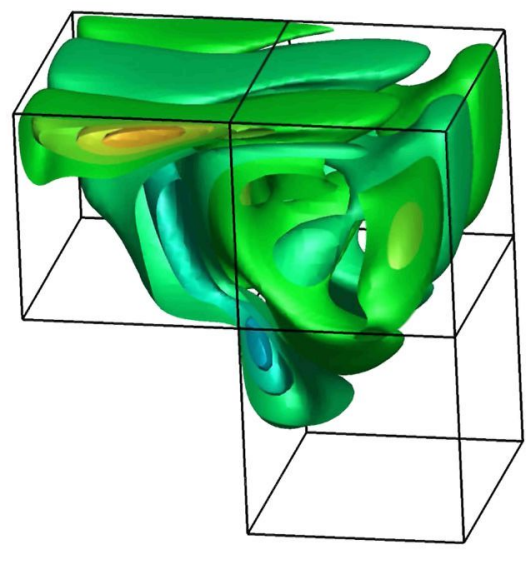

Figure 2. BiGlobal instability analysis of an L-shaped cavity. (a) Growth rate $\left(\lambda_{r}\right)$ of the most unstable 3D mode for two Reynolds 900 and 1100 and varying non-dimensional spanwise length $1<\beta<30$ (arrows show selected spanwise wavenumbers) and (b) Spanwise velocity component of the unstable BiGlobal eigenmode for $\operatorname{Re}=900$ and $\beta=10$.

leading to the 3D modes of relatively short wave length is associated to a centrifugal type instability [43]. We note that to obtain a base flow for the cavity simulations is suffices to run a two-dimensional calculation inhibiting the $w$-velocity flow component.

Preliminary studies for a range of Reynolds and spanwise wavenumbers were performed by the second author using BiGlobal analysis [8]. This preliminary work has enabled the selection of appropriate flow conditions to compare the various techniques, when one or two 3D unstable modes are present in the cavity. Figure 2.(a) shows the variation of the growth rates $\left(\lambda_{r}\right)$ for two Reynolds numbers and a range of spanwise wavenumbers $\left(\beta=2 \pi / L_{z}\right.$, where $L_{z}$ is the cavity length in the $z$-direction). Note that $\lambda_{r}>0$ characterises unstable modes.

Two flow conditions have been retained for the present work. In the first case, $\operatorname{Re}=900$ and $\beta=10$ (resp. $L_{z}=0.628$ ), only one 3D instability develops and in the second case, $\operatorname{Re}=1100$ and $\beta=22.5$ (resp. $L_{z}=0.279$ ), two 3D instabilities grow with time. In our calculations, the Reynolds number is calculated using the characteristic lid velocity $U=1$ and length $L=1$. Vertical arrows in 2.(a) show the two selected spanwise wavenumbers used in the analysis. In addition, 2.(b) shows the shape of the unstable 3D $w$-component of the eigenmode found using the matrix-forming BiGlobal approach at $\operatorname{Re}=900$ and $\beta=10$, which is to be compared to the eigenmodes provided by other techniques in the following section.

\subsection{Instability at $R e=900$ and $\beta=10$ : one $3 D$ unstable mode}

The L-shaped cavity at this Reynolds number shows one unique 3D unstable mode. The shape of the spanwise velocity component of the unstable eigenmode, obtained using the BiGlobal approach, was shown in Figure 2.(b) and can be compared to non-linear and linearised DNS simulations shown in Figure 3.(a) and Figure 3.(b). The shapes of the 3D instabilities that are visualised through velocity isocontours of $w$-velocity compare favourably to the BiGlobal mode. The visualisation of the DNS structures is possible in this case since the base flow has zero $w$-velocity component and there is only one unstable mode present in the simulation. In addition, the three-dimensional modes (only real part is shown) obtained using the DMD technique applied to non-linear and linear DNS results, Figure 3.(c) and 3.(d), may be compared to the DNS structures (Figure 3.(a)) and the BiGlobal eigenmode (Figure 2.(b)) showing good agreement.

Quantitative results for the dynamical information is summarised in Table I. We observe that all methods compare well, hence cross-validating the various methodologies presented. Note that the results from DNS simulations (non-linear and linearised solvers) have been obtained by curve fitting and Fourier analysis of the time evolving $w$-velocity (shown in Figure 1.(b)) and lead to the growth 
(a)

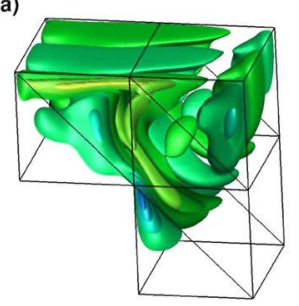

(b)

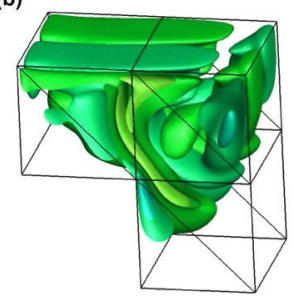

(c)

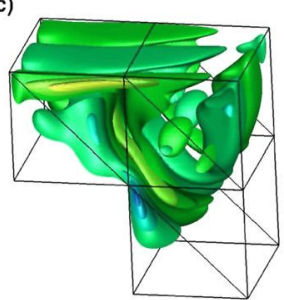

(d)

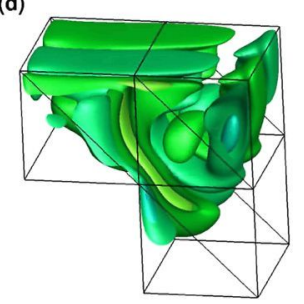

Figure 3. 3D global instability analysis of an L-shaped cavity at Reynolds 900 and non-dimensional spanwise length of 0.628 , simulation with polynomial order $\mathrm{k}=17$ in the $\mathrm{x}-\mathrm{y}$ plane and 64 Fourier planes in the $z$-direction: (a) Nonlinear DNS (NDNS): 10 iso-surfaces of $z$ velocity, (b) Linearised DNS ( $\mathcal{L}$ DNS): 10 isosurfaces of $w$-velocity, (c) $\mathcal{N}$ DNS+DMD: real part of the most unstable mode and (d) $\mathcal{L}$ DNS+DMD: real part of the most unstable mode.

Table I. L-shaped cavity at Reynolds number $R e=900$ and $\beta=10$, growth rate $\lambda_{r}$ and frequency $\lambda_{i}$ of the three-dimensional unstable mode. Non-linear and linear DNS results are compared to linear BiGlobal analysis and DMD results from non-linear ( $\mathcal{N D N S}$ ) and linear $(\mathcal{L D N S})$ simulations.

\begin{tabular}{lcc}
\hline & $\lambda_{r}$ & $\lambda_{i}$ \\
\hline $\mathcal{N}$ DNS & 0.1025 & 1.0295 \\
$\mathcal{L}$ DNS & 0.1069 & 1.0295 \\
BiGlobal & 0.1023 & 1.0284 \\
$\mathcal{N}$ DNS+DMD & 0.1047 & 1.0303 \\
$\mathcal{L}$ DNS+DMD & 0.1167 & 1.0387 \\
\hline
\end{tabular}

rates and frequencies summarised in the table. Results from non-linear DNS simulations compare well with the eigenvalues obtained from BiGlobal analysis. In addition, the table shows that both non-linear and linearised solvers can predict the growth rate and frequency of the three-dimensional mode. The DMD analysis provides very similar results if used in combination with non-linear or linearised solvers, showing that, as long as the snapshots are taken within the exponential growth zone, linearisation is not necessary. However, let us note that the DMD analysis, when using nonlinear simulations, might become difficult to perform if far from the bifurcation point. Our analysis assumes that it is possible to obtain snapshots during the exponential growth of a small perturbation introduced in the simulation. If the flow conditions are too far from equilibrium, it is likely that the flow saturates almost instantly not providing enough time to sample the exponential growth region for DMD analysis.

\subsection{Instabilities at $\operatorname{Re}=1100$ and $\beta=22.5$ : two $3 D$ unstable modes}

In this section, we push the previous comparison forward to a flow regime where two unstable 3D modes develop. Table II compares growth rates and frequencies obtained using the BiGlobal instability analysis together with DMD results combined with the non-linear and linearised DNS solvers. Results for all methods compare favourably. Differences in the growth rates provided by the BiGlobal and the DMD analysis may be explain by the very different underlying numerics used to compute these cases: matrix forming Chebyshev spectral collocation and time-stepping discontinuous Galerkin methods. These techniques are different when computing both the base flows and the perturbation evolution.

Let us note that in this case, where two unstable modes are present, the direct computation of growth rates and frequencies from the DNS simulations is more difficult, since the contributions of various modes overlap. It is in these cases, where the DMD analysis proves advantageous to extract the dominant structures for complex 3D flow. In addition, the eigenmodes issued from BiGlobal 
Table II. L-shaped cavity at Reynolds number $R e=$ 1100 and $\beta=22.5$, growth rate $\lambda_{r}$ and frequency $\lambda_{i}$ of the three-dimensional two unstable modes. Linear BiGlobal analysis and DMD results from non-linear $(\mathcal{N D N S}+\mathrm{DMD})$ and linear $(\mathcal{L} \mathrm{DNS}+\mathrm{DMD})$ simulations.

\begin{tabular}{lcclccc}
\hline & \multicolumn{2}{c}{ Mode 1 } & & \multicolumn{2}{c}{ Mode 2 } \\
\cline { 2 - 3 } \cline { 6 - 7 } & $\lambda_{r}$ & $\lambda_{i}$ & & $\lambda_{r}$ & $\lambda_{i}$ \\
\hline BiGlobal & 0.0789 & 0.0000 & & 0.0617 & 1.0899 \\
$\mathcal{N}$ DDNS+DMD & 0.0926 & 0.0000 & & 0.0786 & 1.0864 \\
$\mathcal{L}$ DNS+DMD & 0.1001 & 0.0000 & & 0.0796 & 1.0883 \\
\hline
\end{tabular}
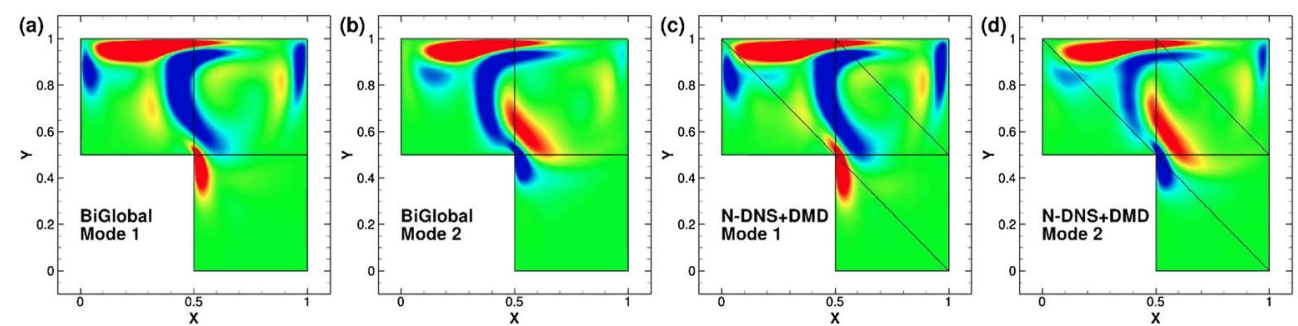

Figure 4. 3D global instability analysis of an L-shaped cavity at Reynolds 1100 and non-dimensional spanwise length of 22.5: (a) Unstable BiGlobal eigenmode 1, (b) Unstable BiGlobal eigenmode 2, (c) Non-linear DNS + DMD: Unstable eigenmode 1 and (d) Non-linear DNS + DMD: Unstable eigenmode 2. All cases show a spanwise slice of the $w$-velocity component through the $3 \mathrm{D}$ modes.

analysis and non-linear DNS analysed with the DMD technique are depicted in Figure 4, and show to be in good agreement, hence validating the methodology when various unstable modes evolve with time.

\section{ADJOINT PROBLEM AND SENSITIVITY}

In this section, we obtain 3D adjoint modes; i.e. modes associated to the linearised adjoint NS equations described in Section 2. More specifically, we time-step the linearised adjoint NS equations in time to produce snapshots that are subsequently analysed using the DMD technique. Adjoint modes are obtained for the L-shaped cavity for the first set of flow parameters, $\mathrm{Re}=900$ and $\beta=10$ (as in Section 3.1). In this work and to the best of our knowledge, we present novel results for 3D adjoint modes and sensitivity maps using the DMD technique.

Adjoint modes are essential to provide insight into flow control to receptivity to external mass and momentum forcing $[4,5]$. In addition, with the adjoint modes at hand, it is easy to compute the sensitivity of the flow to local feedback (i.e. structural sensitivity defining the wavemaker region) [23] and the sensitivity to base flow modifications [24].

On the one hand, the structural sensitivity can be directly computed using the direct and adjoint modes through the relationship: $S=\left\|\mathbf{u}^{*}\right\| \cdot\left\|\mathbf{u}^{\prime}\right\|$ with $<\mathbf{u}^{*}, \mathbf{u}^{\prime}>=1$, where $\mathbf{u}^{\prime}$ and $\mathbf{u}^{*}$ are the direct and adjoint velocity modes and $<\bullet, \bullet>$ denotes the $L_{2}$ inner product with associated norm $\|\bullet\|=\langle\bullet, \bullet\rangle^{1 / 2}$. This sensitivity locates the zones were direct and adjoint modes overlap and reveals the flow regions were a localised force would produce the largest drift in the eigenvalues (sometimes referred to as wavemaker region, see Giannetti and Luchini [23] for further details). On the other hand, the growth rate sensitivity to a base flow modification can be computed using:

$$
\nabla_{\overline{\mathbf{u}}}^{B} \lambda=-\left[\nabla \mathbf{u}^{\prime}\right]^{H} \cdot \mathbf{u}^{*}+\nabla \mathbf{u}^{*} \cdot \mathbf{u}^{\prime},
$$

with the normalisation condition $<\mathbf{u}^{*}, \mathbf{u}^{\prime}>=1$, as shown by Marquet et al. [24] and provides the spatial location where a small modification of the base flow provides the largest eigenvalue drift. In addition, the base flow sensitivity, provides the gradients of the sensitivity, which can 

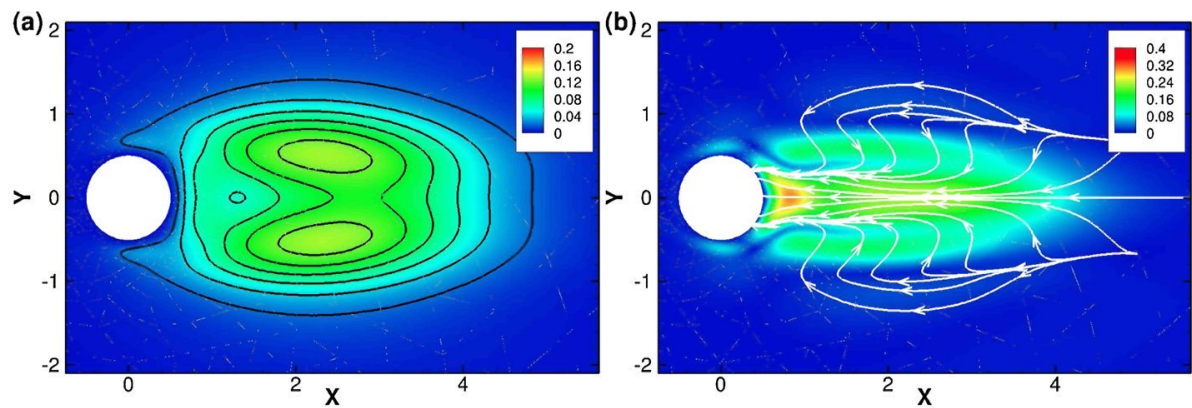

Figure 5. Circular cylinder at $\mathrm{Re}=45$ : (a) Structural sensitivity and (b) Growth rate sensitivity to base flow modification.

Table III. Non-dimensional frequency $\left(\right.$ frequency $\left.=\frac{\lambda_{i}}{2 \pi}\right)$ for the least stable eigenvalue for the circular cylinder at $\operatorname{Re}=45$.

\begin{tabular}{lc}
\hline Reference & frequency \\
\hline Giannetti and Luchini [23] & 0.118 \\
Marquet et al. [24] & 0.116 \\
Crouch et al. [45] & 0.115 \\
Present: $\mathcal{L}$ DNS+DMD & 0.118 \\
\hline
\end{tabular}

be represented using streamlines (see Figure 5.(b)) and give information on the direction of the base flow modification that results in a stabilising or destabilising effect in the eigenvalue growth rate.

\subsection{Validation for a $2 D$ cylinder flow at $R e=45$}

To validate our methodology, we compute the sensitivity to local feedback (i.e. structural sensitivity or wavemaker region) and the sensitivity of the growth rate to a modification of the base flow, for the 2D flow after a circular cylinder at $\mathrm{Re}=45$. The critical Reynolds numbers for the circular cylinder is $\operatorname{Re}_{\text {crit }} \approx 46.6$ (see for example [14, 23]), where a Hopf type bifurcation is responsible of a 2D unstable regime leading to a von Kármán type vortex street. For steady regimes (i.e. $\operatorname{Re}<\operatorname{Re}_{\text {crit }}$ ) the flow is steady and hence the base flow is naturally provided by advancing the unsteady NS equations in time. There is no requirement of any numerical technique to obtain a steady base flow (e.g. Newton method or selective frequency damping [44]) .

The DMD technique is used to extract direct and adjoint modes from the linearised and adjoint linearised snapshots produced by the discontinuous Galerkin solver, which are subsequently used to calculate the structural sensitivity and the sensitivity to base flow modification shown in Figure 5.(a) and 5.(b), respectively. These results compare well with previously published results [23, 24], validating the methodology.

In addition, Table III summarises previous published values $[23,24,45]$ for the frequency of the least stable eigenmode for the circular cylinder case at $\mathrm{Re}=45$. We observe excellent agreement of the DMD mode extracted from linearised NS equations when compared to published results.

\section{2. $3 D$ sensitivities for the $L$-shape cavity at $R e=900$ and $\beta=10$}

Having validated the procedure, we note that no significant changes, other than the computational cost, are introduced when considering 3D flows. We proceed to study the 3D sensitivities for the $\mathrm{L}$-shaped cavity at $\mathrm{Re}=900$ and $\beta=10$.

To the authors knowledge, these sensitivity maps are given for the first time in this work. Figure 6 shows the 3D direct, adjoint modes (real parts only) together with the associated structural sensitivity (the latter computed using real and imaginary information). 
(a)

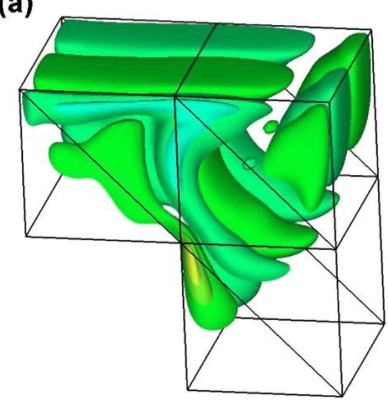

(b)

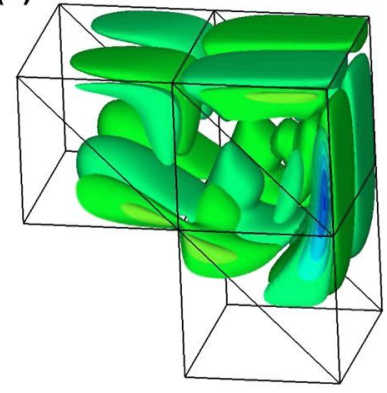

(c)

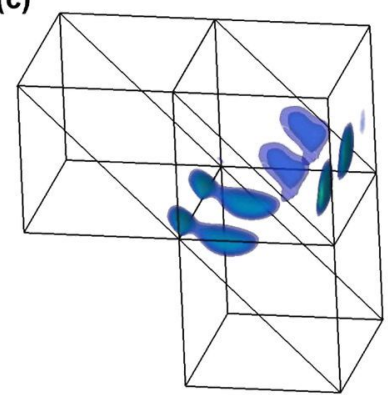

Figure 6. L-shaped cavity at Reynolds 900 with a non-dimensional spanwise length of 0.628 , showing 10 iso-surfaces: (a) Real part of the direct mode (b) Real part of the adjoint mode and (c) Structural sensitivity.
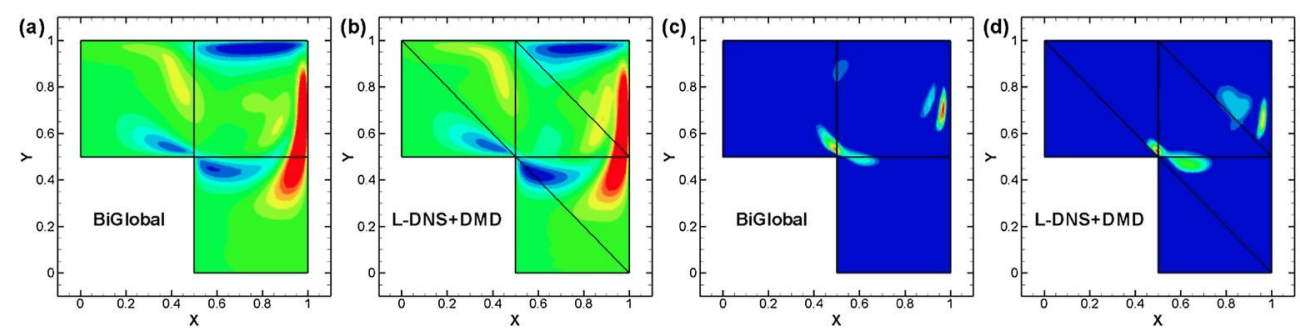

Figure 7. L-shaped cavity at Reynolds 900 with a non-dimensional spanwise length of 0.628: (a) Real part of the BiGlobal adjoint mode, (b) Real part of the $\mathcal{L}$ DNS+DMD adjoint mode, (c) BiGlobal structural sensitivity and (d) $\mathcal{L}$ DNS+DMD structural sensitivity. All cases show a spanwise slice.

Inspection of Figure 6.(c) reveals three pairs of regions of high sensitivity located within the main recirculation of the 2D base flow (see Figure 1). In particular, the highest levels appear close to the L-shape corner within two distinct lobes separated by the 3D mode wavelength (i.e. $L_{\text {lobe }} \sim \frac{1}{2} L_{z}$ ).

In addition and for the sake of completeness, we compare the adjoint modes corresponding to the most unstable direct modes in Figure 7.(a) and 7.(b), and the associated structural sensitivity, in Figure 7.(c) and 7.(d). These results have been obtained using the continuous BiGlobal approach and the DMD technique together with the time-stepped adjoint linearised NS equations. The figures show that very good agreement is obtained between matrix forming and matrix free methods.

To gain insight into the sensitivity of these instabilities, we determine the sensitivity to modifications of the base flow. Figure 8.(a) shows 3D iso-surfaces of the most sensitive flow regions to base flow modifications and is detailed in Figure 8.(b) for a particular $z$-slice providing a $2 \mathrm{D}$ contour of sensitivity. In addition, Figure 8.(c) depicts 2D streamlines for the gradients of the sensitivity to a modification of the base flow. Both sensitivities, Figure 6.(c) and Figure 8.(a), provide regions of sensitivity that are high near the L-shaped corner. It can be seen that the sensitivity to a modification of the base flow, Figure 8.(b), follows the primary circulation pattern of the 2D base flow as shown in Figure 1.(a). However, the streamlines shown in Figure 8.(c) do not provide a clear indication on the direction in which the base flow should be modified. It may be speculated that modifications of the main recirculation region together with its interaction with the L-shape corner (i.e. shear layer region) may affect the $3 \mathrm{D}$ instability. To explore this possibility, we consider a modified version of the L-shaped cavity.

\section{A GEOMETRIC MODIFICATION OF THE L-SHAPED CAVITY}

The previous section showed that a modification of the flow near the L-shaped corner of the cavity may provide the highest stabilising (or destabilising) effect in terms of the 3D instability for Re $=900$ and $\beta=10$ (i.e. $L_{z}=0.628$ ).

In this section, we propose a modified shape (rounded corner) for the L-shaped cavity which aims at decreasing the growth rate of the 3D instability. Figure 9.(a) and 9.(b) show the resulting 
(a)

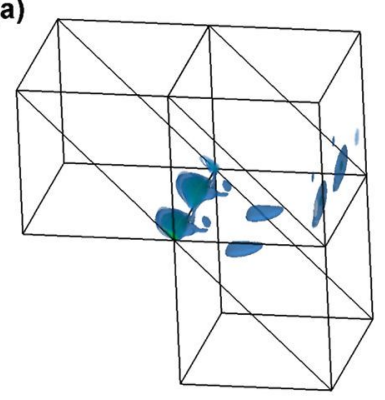

(b)

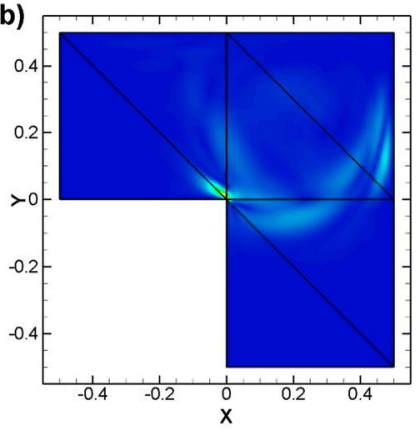

(c)

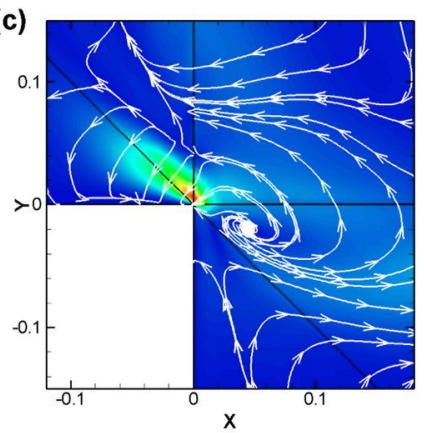

Figure 8. L-shaped cavity at Reynolds 900 with a non-dimensional spanwise length of 0.628 and growth rate sensitivity to base flow modification: (a) 3D iso-surfaces (10 levels shown), (b) 3D streamlines, (c) Contours at section $z=0.24\left(z / L_{z}=0.38\right)$ and (d) $2 \mathrm{D}$ Streamlines at at section $z=0.24\left(z / L_{z}=0.38\right)$.

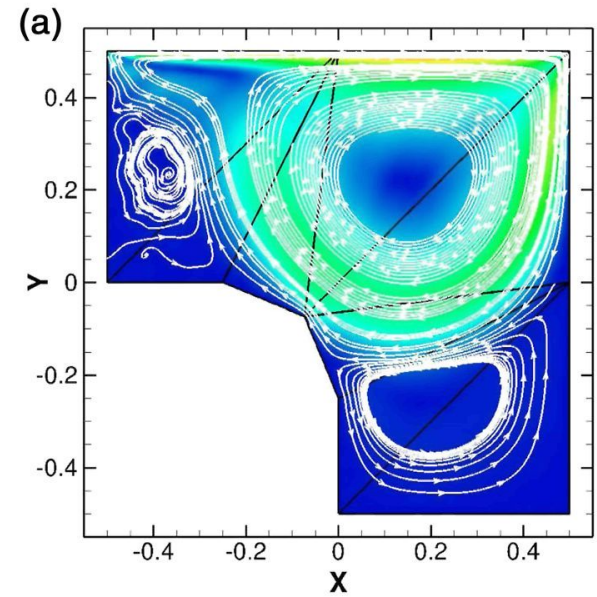

(b)

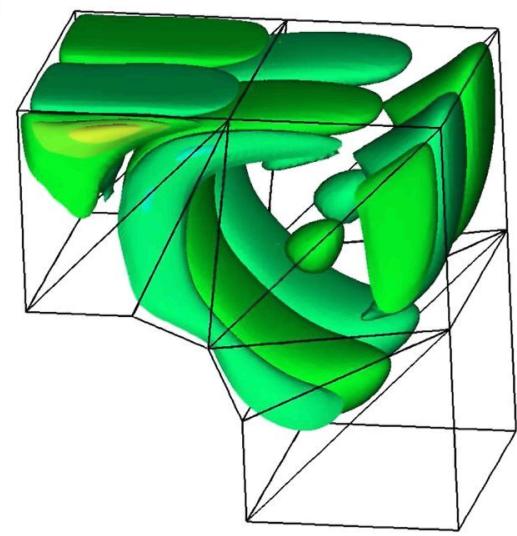

Figure 9. Modified L-shaped cavity at Reynolds 900 with a non-dimensional spanwise length of 0.628 . (a) 2D base flow: 2D streamlines and velocity magnitude contours and (b) 3D instability issued form nonlinear DNS.

2D base flow and iso-contours for the 3D instability, respectively, issued from a non-linear DNS simulations for the modified cavity. The 3D instability associated to this new geometry has a growth rate $\lambda_{r}=0.0537$ and frequency $\lambda_{i}=0.0745$. The growth rate and frequency for the $3 \mathrm{D}$ instability before modification of the geometry, when evaluated from the non-linear DNS solution, were detailed in Section $3.1 \lambda_{r}=0.1025$ and $\lambda_{i}=1.0295$. We observe that the modified geometry reduces the growth rate by a factor of two and decreases its frequency. It may be concluded that this new geometry is more stable, in terms of 3D instabilities, for this particular Reynolds number and spanwise length that the original cavity, showing the potential of the presented methodology. This modified geometry aimed to show the potential of the methodology introduce in this work but does not represent the optimum shape. To obtain an optimal shape further studies would be required.

\section{CONCLUSIONS}

This work provides comparisons of high-order numerical techniques, including high-order continuous and discontinuous Galerkin formulations, to predict 3D global instability analysis and sensitivity maps at an affordable computational cost for general 3D complex geometries. These methods have been applied to characterise 3D instabilities arising within a lid driven L-shaped cavity at various Reynolds numbers and spanwise lengths. 
The work presented has shown that the relatively new high-order numerical approach to solve the incompressible NS equations based on a discontinuous Galerkin variational formulation is able to predict the onset of 3D flow stabilities through DNS calculations. In addition, the recently developed DMD technique, has shown to be an efficient, robust and cost effective mean to extract dynamical information from snapshots produced by DNS computations. It has been shown that the modes extracted from non-linear solvers (no need for linearisation) agree well with matrix forming (i.e. BiGlobal) and matrix-free linearised methods (i.e. linearised time marched NS). The latter remark expands the potential of this type of analysis based on snapshot analysis to existing Computational Fluid Dynamics codes, without need of modifications. Furthermore, since non-linear calculations are performed and resulting snapshots analysed using the DMD technique directly there is no need to obtain a base flow.

The methodology presented shows that it is possible to obtain an approximation of the 3D direct and adjoint modes, as well as the associated sensitivity fields (e.g. to local feedback and to base flow modifications), at a reduced computational cost for general complex 3D geometries. This knowledge may be used to provide valuable information of the most sensitive flow regions that may help control or attenuate 3D instabilities. The potential for finding more stable geometries is illustrated through a modification of the L-shape cavity. Finally, this paper provides physical global instability and sensitivity results for the lid driven L-shaped cavity that have not been reported before.

\section{ACKNOWLEDGEMENTS}

EF and EV would like to thank the European Commission for the financial support of the ANADE project (Advances in Numerical and Analytical tools for DEtached flow prediction) under grant contract PITN-GA-289428.

\section{REFERENCES}

1. Schmid P, Henningson D. Stability and Transition in Shear Flows, Applied Mathematical Science, vol. 142. Springer-Verlag, New York Inc., 2001.

2. Theofilis V. Global linear instability. Annual Review of Fluid Mechanics 2011; 43(1):319-352.

3. Sipp D, Barbagallo A, Marquet O, Meliga P. Dynamics and control of global instabilities in open-flows: A linearized approach. Applied Mechanics Reviews 2010; 63(3):030801.

4. Chomaz J. Global instabilities in spatially developing flows: Non-normality and nonlinearity. Annual Review of Fluid Mechanics 2005; 37(1):357-392.

5. Luchini P, Bottaro A. Adjoint equations in stability analysis. Annual Review of Fluid Mechanics 2014; 46(1): 493-517.

6. Huerre P, Monkewitz P. Local and global instabilities in spatially developing flows. Annual Review of Fluid Mechanics 1990; 22(1):473-537.

7. Bagueri S, Schlatter P, Schmid P, Henningson D. Global stability of a jet in crossflow. Joumal of Fluid Mechanics 20094; 624:33-44.

8. Vicente JD. Spectral multidomain methods for global instability analysis of complex cavity flows. Ph.D. Thesis, School Aeronautics, ETSIA-UPM, Madrid, Spain, 2010.

9. Edwards W, Tuckerman L, Friesner R, Sorensen D. Krylov methods for the incompressible Navier-Stokes equations. Journal of Computational Physics 1994; 110(1):82-102.

10. Tuckerman L, Barkley D. Bifurcation analysis for timesteppers. In Numerical Methods for Bifurcation Problems and Large-Scale Dynamical Systems, vol. 119, Doedel E, Tuckerman L (eds)., IMA Volumes in Mathematics and its Applications. Springer: New York, 2000; 452-466.

11. Knoll D, Keyes D. Jacobian-free Newton-Krylov methods: a survey of approaches and applications. Journal of Computational Physics 2004; 193(2):357-397.

12. Bagheri S, Akervik E, Brandt L, Henningson D. Matrix-free methods for the stability and control of boundary layers. AIAA Journal 2009; 47(5):1057-1068.

13. Barkley D, Blackburn H, Sherwin S. Direct optimal growth analysis for timesteppers. International Journal for Numerical Methods in Fluids 2008; 57(9): 1435-1458.

14. Bagheri S. Koopman-mode decomposition of the cylinder wake. Journal of Fluid Mechanics 20137; 726:596-623.

15. Monokrousos A, Åkervik E, Brandt L, Henningson D. Global three-dimensional optimal disturbances in the blasius boundary-layer flow using time-steppers. Journal of Fluid Mechanics 20105; 650:181-214.

16. Cherubini S, Robinet J, Bottaro A, De Palma P. Optimal wave packets in a boundary layer and initial phases of a turbulent spot. Journal of Fluid Mechanics 20108; 656:231-259.

17. Pringle C, Kerswell R. Using nonlinear transient growth to construct the minimal seed for shear flow turbulence. Physical Review Letters 2010; 105:154502. 
18. Monokrousos A, Bottaro A, Brandt L, Di Vita A, Henningson D. Nonequilibrium thermodynamics and the optimal path to turbulence in shear flows. Physical Review Letters 2011; 106:134502.

19. Cherubini S, De Palma P, Robinet J, Bottaro A. The minimal seed of turbulent transition in the boundary layer. Journal of Fluid Mechanics 201112; 689:221-253.

20. Berkooz G, Holmes P, Lumley J. The proper orthogonal decomposition in the analysis of turbulent flows. Annual Review of Fluid Mechanics 1993; 25(1):539-575.

21. Mezić I. Analysis of fluid flows via spectral properties of the Koopman operator. Annual Review of Fluid Mechanics $2013 ; 45(1): 357-378$.

22. Schmid P. Dynamic mode decomposition of numerical and experimental data. Journal of Fluid Mechanics 2010; 656:5-28.

23. Giannetti F, Luchini P. Structural sensitivity of the first instability of the cylinder wake. Journal of Fluid Mechanics 20076; 581:167-197.

24. Marquet O, Sipp D, Jacquin L. Sensitivity analysis and passive control of cylinder flow. Journal of Fluid Mechanics 200811; 615:221-252.

25. Karniadakis G, Sherwin S. Spectral/hp Element Methods for Computational Fluid Dynamics. Oxford Science Publications: Oxford, United Kingdom, 2005.

26. Barkley D, Henderson R. Three-dimensional Floquet stability analysis of the wake of a circular cylinder. Journal of Fluid Mechanics 1996; 322:215-241.

27. Ferrer E, Willden R. A high order discontinuous Galerkin-Fourier incompressible 3 d Navier-Stokes solver with rotating sliding meshes. Journal of Computational Physics 2012; 231(21):7037-7056.

28. Ferrer E. A high order discontinuous Galerkin-Fourier incompressible $3 \mathrm{~d}$ Navier-Stokes solver with rotating sliding meshes for simulating cross-flow turbines. Ph.D. Thesis, University of Oxford, United Kingdom, 2012.

29. Ferrer E, Willden R. A high order discontinuous Galerkin finite element solver for the incompressible Navier-Stokes equations. Computers \& Fluids 2011; 46(1):224-230.

30. Chen K, Tu J, Rowley C. Variants of dynamic mode decomposition: Boundary condition, Koopman, and Fourier analyses. Journal of Nonlinear Science 2012; 22(6):887-915.

31. Rowley C, Mezia I, Bagheri S, Schlatter P, Henningson D. Spectral analysis of nonlinear flows. Journal of Fluid Mechanics 200912; 641:115-127.

32. Duke D, Soria J, Honnery D. An error analysis of the dynamic mode decomposition. Experiments in Fluids 2012; 52(2):529-542.

33. Jovanović M, Schmid P, Nichols J. Sparsity-promoting dynamic mode decomposition. Physics of Fluids 2014; 26(2):024103.

34. Saad Y. Numerical Methods for Large Eigenvalue Problems. Manchester University Press: Manchester, 1992.

35. Shankar P, Deshpande M. Fluid mechanics in the driven cavity. Annual Review of Fluid Mechanics 2000; 32(1): 93-136.

36. Oosterlee C, Wesseling P, Segal A, Brakkee E. Benchmark solutions for the incompressible Navier-Stokes equations in general coordinates on staggered grids. International Joumal of Numerical Methods in Fluids 1993; 17(4): 301-321.

37. Perng C, Street R. A coupled multigrid-domain-splitting technique for simulating incompressible flows in geometrically complex domains. International Journal for Numerical Methods in Fluids 1991; 13(3):269-286.

38. Deliceoğlu A, Aydın S. Flow bifurcation and eddy genesis in an l-shaped cavity. Computers \& Fluids 2013; 73(0): 24-46.

39. Deliceoğlu A, Aydın S. Topological flow structures in an l-shaped cavity with horizontal motion of the upper lid. Journal of Computational and Applied Mathematics 2014; 259, Part B(0):937-943.

40. Giannetti F, Luchini P, Marino L. Characterization of the three-dimensional instability in a lid-driven cavity by an adjoint based analysis. In Seventh IUTAM Symposium on Laminar-Turbulent Transition, Vol. 18. Springer: Netherlands, 2010; 165-170.

41. Basley J, Pastur L, Delprat N, Lusseyran F. Space-time aspects of a three-dimensional multi-modulated open cavity flow. Physics of Fluids 2013; 25:064105.

42. Bres GA, Colonius T. Three-dimensional instabilities in compressible flows over open cavities. Journal of Fluid Mechanics 2008; 599:309-339.

43. Albensoeder S, Kuhlmann HC, Rath HJ. Three-dimensional centrifugal-flow instabilities in the lid-driven-cavity problem. Physics of Fluids (1994-present) 2001; 13(1):121-135.

44. Åkervik E, Brandt L, Henningson D, Hopffner J, Marxen O, Schlatter P. Steady solutions of the Navier-Stokes equations by selective frequency damping. Physics of Fluids 2006; 18(6):068102.

45. Crouch J, Garbaruk A, Magidov D. Predicting the onset of flow unsteadiness based on global instability. Journal of Computational Physics 2007; 224(2):924 -940. 